\title{
How effective is an in-hospital heart failure self-care program in a Japanese setting? Lessons from a randomized controlled pilot study
}

This article was published in the following Dove Press journal:

Patient Preference and Adherence

18 February 2016

Number of times this article has been viewed

\author{
Naoko P Kato ${ }^{1-3}$ \\ Koichiro Kinugawa' \\ Miho Sano ${ }^{4}$ \\ Asuka Kogure 4 \\ Fumika Sakuragi ${ }^{5}$ \\ Kihoko Kobukata ${ }^{4}$ \\ Hiroshi Ohtsu ${ }^{6}$ \\ Sanae Wakita ${ }^{4}$ \\ Tiny Jaarsma ${ }^{2}$ \\ Keiko Kazuma ${ }^{7}$
}

'Department of Therapeutic Strategy for Heart Failure, The University of Tokyo Graduate School of Medicine, Tokyo, Japan; ${ }^{2}$ Department of Social and Welfare Studies, Faculty of Health Sciences, Linköping University, Linköping, Sweden; ${ }^{3}$ JSPS Postdoctoral Fellow for Research Abroad, Tokyo, ${ }^{4}$ Department of Nursing, ${ }^{5}$ Department of Pharmacy, The University of Tokyo Hospital, ${ }^{6}$ Department of Clinical Data Management, ${ }^{7}$ Department of Adult Nursing, The University of Tokyo Graduate School of Medicine, Tokyo, Japan
Correspondence: Naoko P Kato Department of Therapeutic Strategy for Heart Failure, The University of Tokyo Graduate School of Medicine, 7-3-I, Hongo, Bunkyo-ku, Tokyo I I38655, Japan

Tel +8I 358009082

$\mathrm{Fax}+81358009082$

Email naokat-tky@umin.ac.jp
Background: Although the effectiveness of heart failure (HF) disease management programs has been established in Western countries, to date there have been no such programs in Japan. These programs may have different effectiveness due to differences in health care organization and possible cultural differences with regard to self-care. Therefore, the purpose of this study was to evaluate the effectiveness of a pilot HF program in a Japanese setting.

Methods: We developed an HF program focused on enhancing patient self-care before hospital discharge. Patients were randomized 1:1 to receive the new HF program or usual care. The primary outcome was self-care behavior as assessed by the European Heart Failure SelfCare Behavior Scale (EHFScBS). Secondary outcomes included HF knowledge and the 2-year rate of HF hospitalization and/or cardiac death.

Results: A total of 32 patients were enrolled (mean age, 63 years; 31\% female). There was no difference in the total score of the EHFScBS between the two groups. One specific behavior score regarding a low-salt diet significantly improved compared with baseline in the intervention group. HF knowledge in the intervention group tended to improve more over 6 months than in the control group (a group-by-time effect, $F=2.47, P=0.098$ ). During a 2-year follow-up, the HF program was related to better outcomes regarding HF hospitalization and/or cardiac death (14\% vs 48\%, log-rank test $P=0.04)$. In Cox regression analysis after adjustment for age, sex, and logarithmic of B-type natriuretic peptide, the program was associated with a reduction in HF hospitalization and/or cardiac death (hazard ratio, $0.17 ; 95 \%$ confidence interval, $0.03-0.90 ; P=0.04$ ).

Conclusion: The HF program was likely to increase patients' HF knowledge, change their behavior regarding a low-salt diet, and reduce HF hospitalization and/or cardiac events. Further improvement focused on the transition of knowledge to self-care behavior is necessary.

Keywords: discharge education, disease management, hospitalization, knowledge, nursing, patient education

\section{Introduction}

Heart failure (HF) treatment has remarkably improved over the past 2 decades, but the rate of rehospitalization remains high worldwide, including in Japan, ${ }^{1}$ leading to a considerable burden on the health care system and impairing patients' quality of life. Approximately half of the readmissions for HF are considered to be potentially preventable with adequate self-care and appropriate support. ${ }^{2}$ Although the optimal model of HF management programs is still under debate, ${ }^{3}$ a multidisciplinary HF management program successfully reduces morbidity and mortality in HF patients. ${ }^{4}$ The effects of multidisciplinary HF programs have widely been reported in Western countries, ${ }^{3,5}$ but there have been no such programs in Japan so far. 
One may argue that with the increasing number of meta-analyses and Cochrane reviews with regard to HF management, ${ }^{6,7}$ the time for small trials has passed. However, it should be acknowledged that due to different health care systems, the education of health care professionals, and cultural issues, it is important to test the applicability of programs in non-Western countries. Specifically for Japan, a hospital-based program seems to be the most feasible at present. It is difficult for nurses to provide education for HF patients at an outpatient clinic as Japanese hospitals cannot get reimbursement for this service. ${ }^{8}$ Most HF patients are regularly followed up by their cardiologist or attending physicians under universal health coverage in Japan. Therefore, a multidisciplinary hospital-based program aimed at improving patient self-care seems to be a realistic model to improve patient outcomes in Japan.

HF management programs that focused on enhancing HF self-care of patients are shown to be effective in reducing hospitalizations due to $\mathrm{HF}^{4}{ }^{4}$ An important determinant of self-care adherence is knowledge about HF. ${ }^{9,10} \mathrm{HF}$ patients may have several misunderstandings about HF, which can lead to poor adherence to HF self-care and to the deterioration of HF. ${ }^{11,12}$ There have been a few studies that have investigated the effects of an HF program on both disease-specific knowledge and hospitalization due to HF. ${ }^{13,14}$

The purpose of the present study was to develop an HF self-care program for Japanese patients delivered by a multidisciplinary team before hospital discharge and to evaluate the impact of the program on HF self-care behavior, HF knowledge, and hospitalization for worsening HF. We have also reported lessons learnt from this randomized controlled pilot study.

\section{Methods}

\section{Study design}

This was a single-center, exploratory, randomized controlled pilot study conducted among Japanese patients with HF. Patients were randomized in a 1:1 ratio into a usual care or intervention group and followed up for 2 years. The protocol was approved by the Institutional Review Board on Human Research at the University of Tokyo (No 2145) and registered in the UMIN Clinical Trial Registry system (UMIN000001715, February 18, 2009). All patients provided written informed consent.

\section{Study patients}

Eligible patients were older than 20 years, hospitalized with a primary diagnosis of systolic or diastolic HF. Exclusion criteria were as follows: 1) stage D HF, owing to the low likelihood of benefit from behavioral treatment; 2) New
York Heart Association (NYHA) functional class I because the new HF program targeted patients with HF symptoms; 3) uncertain 12-month prognosis; 4) severe medical or psychiatric comorbid condition; 5) logistical barriers (eg, non-speaking, non-reading Japanese); 6) physician refusal; and 7) patient refusal.

\section{Procedure}

Patients were recruited between 2009 and 2010 in a university hospital at Tokyo. Because this was a pilot study, we set a recruitment period of a year and a follow-up of 2 years. Patients were recruited by four research nurses through inpatient screening. After the baseline examination, one of the research nurses contacted the randomization service office and was sent the treatment assignment. Stratified blocked randomization with regard to age and NYHA class was performed. The patients were blinded to the allocation. A nurse collected data as a researcher. The nurse who collected the data and the nurses who visited patients for the intervention were never the same. Data were collected at baseline and questionnaires were sent at 1 and 6 months after discharge.

\section{HF self-care program}

We developed a multidisciplinary HF management program aimed at improving HF self-care on the basis of the findings from prior studies. ${ }^{4}$ All patients received systematic and planned face-to-face education and counseling from a dietician, a pharmacist, and nurses. The nurses provided the HF education with previously developed materials. ${ }^{15}$ The total time required to provide education was $68 \pm 32$ minutes. ${ }^{15}$ In order to standardize this program, nurse educators were trained through role-play before starting the study. The multidisciplinary team shared a checklist for evaluating the progress of this HF self-care program. Educational topics (Figure 1) included an overview of HF (eg, cardiac function, signs and symptoms, and factors precipitating HF), examination and treatment of HF, self-monitoring (eg, daily weighing), and lifestyle (eg, sodium and fluid intake and physical activity). ${ }^{16}$ Teaching began with the topic that the patient was most interested in. Inspired by the Health Belief Model, ${ }^{17}$ information was provided on why the patient was at risk and how the recommended changes could improve the patient's condition or reduce the risk. To enhance problemsolving skills for recognizing symptom and seeking help, we used a leaflet describing the health care-seeking behavior in three stages: 1) immediately, 2) as soon as possible (within $\sim 1$ week), and 3) upon the next visit to a physician. ${ }^{15}$ A pharmacist provided education about medication (dosing, 


\begin{tabular}{ll}
\hline Category & Example \\
\hline Overall HF & What is cardiac function? \\
& How does the heart work? \\
& What is heart failure? \\
& What are the important signs and symptoms? \\
& What are the factors that worsen HF? \\
& What can we know from a chest X-ray and echocardiography? \\
Examination and treatment & What is BNP? \\
& What are $\beta$-blockers/ACEls/diuretics? \\
Self-monitoring & Which symptoms and signs do I have to monitor? \\
& How do I cope with these symptoms and signs: immediately/as soon as possible/ \\
& upon the next visit to a physician? \\
& How do I check my weight? \\
Lifestyle & Why is a low-salt diet/fluid management/physical activity important? \\
& Why is it important to prevent colds? \\
& Should I stop smoking? Can I drink alcohol? \\
& How do I cope with stress? \\
& Why is it important to see a doctor regularly? \\
\hline
\end{tabular}

Figure I Educational topics in the program.

Abbreviations: HF, heart failure; BNP, B-type natriuretic peptide; ACEl, angiotensin-converting-enzyme inhibitor.

effects of drug, and side effects) and a dietician provided education and counseling with regard to the rationale of salt restriction and how to select and prepare low-salt food.

All patients assigned to the usual care (control group) received standard care and were not provided with structured patient education; however, depending on the individual nurse or physician, the patient received information in writing or orally about medication and lifestyle.

\section{Primary outcome}

The primary outcome was HF self-care behavior, which was assessed by the Japanese version of the European Heart Failure Self-Care Behavior Scale (EHFScBS). ${ }^{18,19}$ This is a 12-item self-administered questionnaire that comprises information on the self-care behavior of HF patients such as daily weighing, fluid restriction, low-salt diet, and medication as well as contacting health care providers on experiencing increased weight gain. For each item, patients rate their selfcare behavior on a five-point scale from 1 ("I completely agree") to 5 ("I do not agree at all"). The total score can range from 12 to 60 , and a higher score indicates poorer self-care behavior. The validity and reliability of the Japanese scale have been confirmed. ${ }^{18}$

\section{Secondary outcomes}

Secondary outcomes were the following: 1) HF knowledge and 2) time to the first HF hospitalization and/or cardiac death. Hospitalization for HF was defined as admission with symptoms or signs of worsening HF. The information on clinical outcomes was collected from medical records and health insurance claims.
Knowledge about HF was assessed using a 15-item Japanese HF knowledge scale, the validity and reliability of which has been confirmed. ${ }^{20}$ The scale evaluates the knowledge of patients regarding HF symptoms, HF-related treatment, and self-care. Scores for each item were summed, giving a range of total scores from 0 to 15 . Higher scores indicate greater knowledge about HF.

\section{Other measures}

The following characteristics of the subjects were recorded: sex, age, marital status, living status, employment, etiology of HF, prior hospitalization for HF, plasma levels of B-type natriuretic peptide, left ventricular ejection fraction, NYHA functional class, comorbidity such as diabetes mellitus and chronic kidney disease (defined as estimated glomerular filtration rate of $<60 \mathrm{~mL} / \mathrm{min} / 1.73 \mathrm{~m}^{2}$ ) calculated using the Japanese formula, and medication. These data were collected from the medical records of patients.

\section{Sample size}

Sample size was based on the assumption that the HF program would produce a 17-point reduction in the EHFScBS score 1 month after discharge. ${ }^{21,22}$ We assumed that the scores at baseline and at 1 month would be 37 and 20 in the intervention program and 37 and 30 in the usual care group, respectively. ${ }^{21,22}$ Assuming a two-sided $\alpha$ of $0.05 \%$ and $80 \%$ power, this led to a sample size of 28 patients eventually distributed between the two treatment groups. The percentage of patients who dropped out of the study was assumed to be $20 \%$; thus, we considered that we needed a total of at least 34 patients in the study. 


\section{Statistical analysis}

Categorical data were presented as frequencies and percentages, and continuous variables were with a normal distribution of the mean and standard deviation. Characteristics of patients were compared using Student's $t$-test or the Mann-Whitney $U$-test for continuous variables, and the chi-square test or Fisher's exact test was used for categorical variables, as appropriate. With respect to B-type natriuretic peptide, we first performed a logarithmic transformation and then analyzed the data using parametric methods.

Linear mixed model was conducted to examine the relationship between groups and changes in the EHFScBS score and the HF knowledge score over 6 months. Fixed effects were groups (intervention vs usual care), time (baseline, 1 and 6 months), and the group-by-time interaction. Unpaired $t$-test was also used to compare these scores between the intervention and usual care groups at each point. To evaluate the differences in these scores within the groups before and after, we used a paired $t$-test for the total score and Wilcoxon signedrank test for the item score. To examine the effects on the endpoint of HF hospitalization and/or cardiac death, KaplanMeier survival curve analysis with log-rank test and Cox proportional-hazard model were used. All analyses for these comparisons between the intervention and usual care groups were conducted according to the originally assigned groups.

The number of missing data for items was estimated with the average score for valid items in the questionnaire. When the missing data accounted for more than $30 \%$ of the questionnaire, we excluded it from the analysis. All statistical tests were two-tailed, and statistical significance was defined as $P<0.05$. All analyses were performed with SAS version 9.3 (SAS Institute Inc., Cary, NC, USA).

\section{Results}

Figure 2 presents the trial profile. Of the 43 patients who were screened, 38 patients were enrolled in the study. Table 1 shows the baseline characteristics of patients. The mean age was 63 years, $31 \%$ were female, $44 \%$ had ischemic etiology, $47 \%$ were newly diagnosed with HF and did not have a history of HF hospitalizations, and $66 \%$ had HF with reduced left ventricular ejection fraction. With regard to comorbidity, $41 \%$ and $69 \%$ had diabetes mellitus and chronic kidney disease, respectively. Approximately $90 \%$ and $80 \%$ of the patients were treated with angiotensin-converting-enzyme inhibitors and/or angiotensin II receptor blockers, and $\beta$-blocker. There were no significant differences in patient characteristics between both the intervention and usual care groups.

\section{HF self-care}

Mean scores of the EHFScBS decreased over time in both the intervention and usual care groups $(F=5.29, P<0.01)$, demonstrating an improvement in HF self-care. Compared with baseline, the EHFScBS score at 1 month after discharge significantly improved in both the intervention (mean \pm standard deviation $[\mathrm{SD}], 36.2 \pm 7.7$ to $30.1 \pm 9.5 ; P=0.04)$ and usual care groups (36.6 \pm 9.5 to $28.5 \pm 9.6 ; P=0.01$; Figure 3 ). However, there were no significant differences of changes in the EHFScBS scores between the two groups over 6 months (a group-by-time interaction, $F=0.44, P=0.65$ ).

With regard to the individual items (Table 2), we found that adhering to daily weighing (item 1) and consuming a low-salt diet (item 9) were significantly improved at 1 month in the intervention group compared with baseline. The improvement on a low-salt diet was also significant at 6 months (item 9). There were no significant differences in changes of scores between the two groups.

\section{HF knowledge}

At baseline, the knowledge score in the intervention group was likely to be higher than in the usual care group (mean $\pm \mathrm{SD} ; 10.6 \pm 3.2$ vs $8.1 \pm 3.6 ; P=0.06$; Figure 4 ). Mean scores on the knowledge scale increased over time in both groups $(F=5.23, P<0.01)$. There was likely to be a group- by-time effect ( $F=2.47, P=0.098$ ), demonstrating that the intervention group tended to improve HF knowledge more than the usual care group over 6 months. The knowledge score in the intervention group was significantly higher than that in the usual care group at 1 month after discharge (13.1 \pm 1.7 vs $8.7 \pm 4.8 ; P=0.03$ ). However, at 6 months, the knowledge score between the groups did not differ significantly (11.5 \pm 2.0 vs $9.7 \pm 2.6, P=0.10)$. Table 3 shows the impacts of the HF program at each time. Compared with the usual care group, the change in scores at 1 month for sodium (item 8) was significantly greater in the intervention group.

\section{Hospitalization for HF/cardiac death}

Of the 32 patients, three patients were excluded from the analysis of clinical outcomes because they were followed up in other hospitals; therefore, we could not collect the medical data. During the median follow-up of 2 years (interquartile range: $0.6-2.0$ ), two patients (14\%) in the intervention group and seven patients (48\%) in the usual care group were hospitalized for worsening HF. During the hospitalization, one patient in the usual care group died from HF. With regarding to all-cause death, 


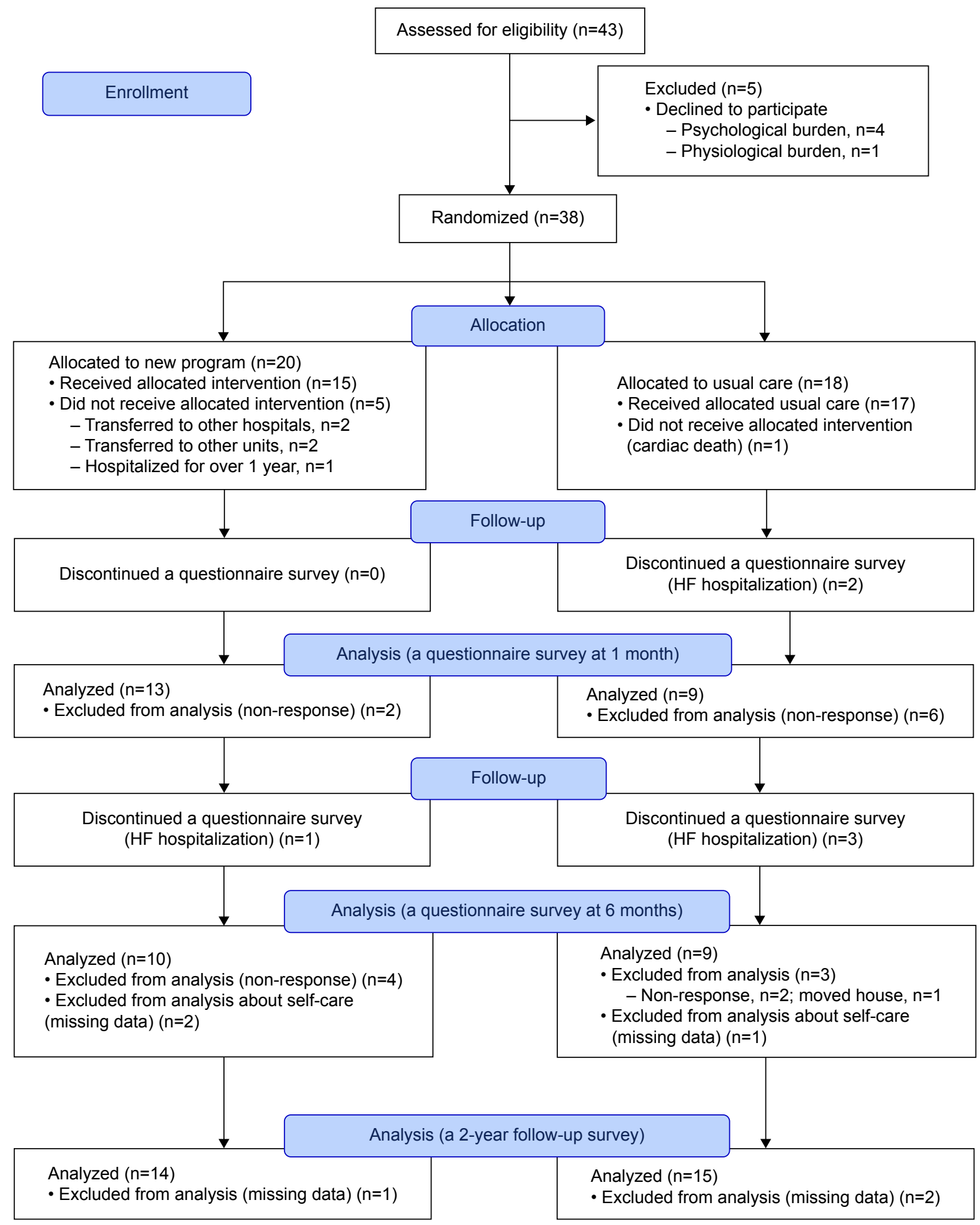

Figure 2 Flowchart of study patients.

Note: Non-response patients did not send back the questionnaire even after 30 days since the day of the survey.

Abbreviation: $\mathrm{HF}$, heart failure.

one patient in the usual care group died from cancer. There was a significant benefit of the HF program on time to the first HF hospitalization/cardiac death (Figure 5; $P=0.04$ ). Cox regression analysis showed that the HF program was likely to reduce HF hospitalization/cardiac death (hazard ratio, $0.23 ; 95 \%$ confidence interval, $0.05-1.09 ; P=0.06$ ). After the adjustment of age, sex, and log B-type natriuretic peptide, the program was still associated with a reduction in 
Table I Characteristics of study patients at baseline

\begin{tabular}{|c|c|c|c|c|}
\hline Characteristics & Total $(n=32)$ & Program $(n=15)$ & Usual care $(n=17)$ & $P$-value \\
\hline \multicolumn{5}{|l|}{ Demographic characteristics } \\
\hline Sex, female & $10(31 \%)$ & $3(20 \%)$ & $7(4 \mid \%)$ & 0.27 \\
\hline Age, years & $63 \pm 14$ & $64 \pm 15$ & $65 \pm 17$ & 0.84 \\
\hline Marital status & & & & 0.11 \\
\hline Single & $6(19 \%)$ & $2(13 \%)$ & $4(24 \%)$ & - \\
\hline Married & 17 (53\%) & II (73\%) & $6(35 \%)$ & - \\
\hline Widowed/divorced & $9(28 \%)$ & $2(13 \%)$ & $7(4 \mid \%)$ & - \\
\hline Education, $\leq 12$ years & 17 (53\%) & $7(47 \%)$ & 10 (59\%) & 0.49 \\
\hline Employment, employed & I5 (47\%) & 7 (47\%) & $8(47 \%)$ & 1.00 \\
\hline Living alone & 14 (44\%) & $5(33 \%)$ & $9(53 \%)$ & 1.00 \\
\hline \multicolumn{5}{|c|}{ Cardiac parameters at discharge } \\
\hline Ischemic etiology & 14 (44\%) & $6(40 \%)$ & $8(47 \%)$ & 0.98 \\
\hline Prior hospitalization for HF & $15(47 \%)$ & $7(47 \%)$ & $8(47 \%)$ & 0.98 \\
\hline NYHA functional class, II & $9(28 \%)$ & $4(27 \%)$ & $5(29 \%)$ & 0.43 \\
\hline BNP, pg/mL, median (QI-Q3) & $238(146-534)$ & $190(|3|-396)$ & $248(183-629)$ & 0.62 \\
\hline LVEF $<50 \%$ & $21(66 \%)$ & $9(60 \%)$ & $12(7 \mid \%)$ & 0.53 \\
\hline Diabetes mellitus & $13(4 \mid \%)$ & $6(40 \%)$ & 7 (4I\%) & 0.95 \\
\hline Chronic kidney disease & $22(69 \%)$ & $10(67 \%)$ & $12(7 \mid \%)$ & 1.00 \\
\hline \multicolumn{5}{|l|}{ Medical therapy } \\
\hline$\beta$-blocker & $26(81 \%)$ & $13(87 \%)$ & $13(76 \%)$ & 0.66 \\
\hline ACEl and/or ARB & 29 (91\%) & $14(93 \%)$ & I5 (88\%) & 1.00 \\
\hline Aldosterone blocker & $20(63 \%)$ & $8(53 \%)$ & $12(7 \mid \%)$ & 0.47 \\
\hline Diuretics & $25(78 \%)$ & $10(67 \%)$ & $15(88 \%)$ & 0.14 \\
\hline \multicolumn{5}{|l|}{ Self-care-related outcomes } \\
\hline EHFScBS score & $36.4 \pm 8.4$ & $36.2 \pm 7.6$ & $36.6 \pm 9.5$ & 0.90 \\
\hline HF knowledge score & $9.4 \pm 3.6$ & $10.6 \pm 3.2$ & $8.1 \pm 3.6$ & 0.06 \\
\hline
\end{tabular}

Notes: Chronic kidney disease was defined as estimated glomerular filtration rate $<60$. Values are $n(\%)$, or mean $\pm S D$.

Abbreviations: HF, heart failure; BNP, B-type natriuretic peptide; LVEF, left ventricular ejection fraction; NYHA, New York Heart Association; ACEl, angiotensinconverting-enzyme inhibitor; ARB, angiotensin II receptor blocker; SD, standard deviation; EHFScBS, European Heart Failure Self-care Behavior Scale.

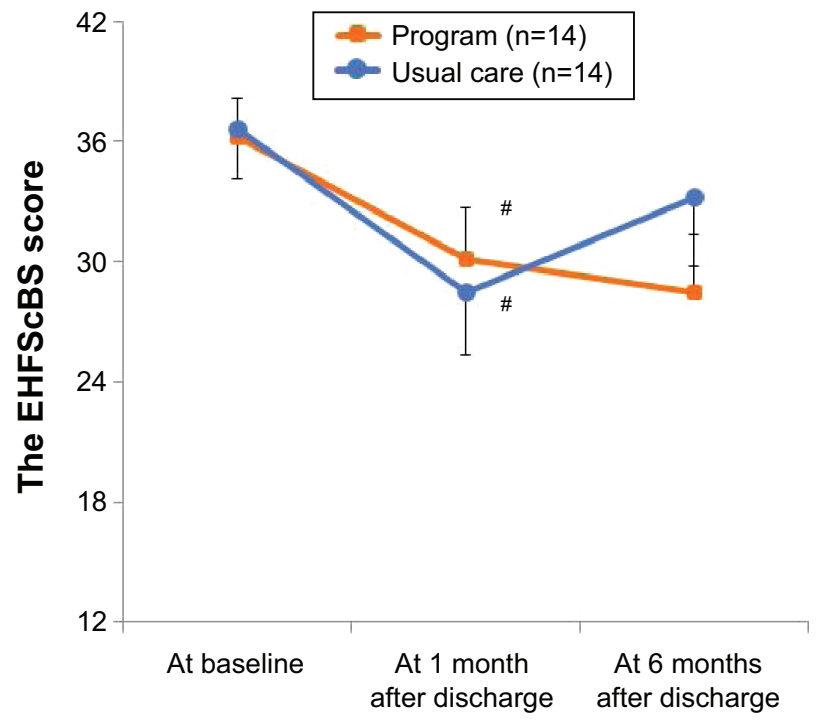

Figure 3 Impacts of the program on heart failure (HF) self-care behavior.

Notes: Data represent mean of the EHFScBS score and error bars represent standard errors of the mean (SEM). Higher score indicates poorer HF self-care (range: $12-60)$. Follow-up time $(F=5.29, P<0.01)$, group $(F=0.05, P=0.83)$, and a group-by-time interaction $(F=0.44, P=0.65)$ by the linear mixed model. ${ }^{*} P<0.05$ by paired $t$-test ( $v s$ baseline).

Abbreviation: EHFScBS, European Heart Failure Self-Care Behavior Scale. cardiac events (hazard ratio, $0.17 ; 95 \%$ confidence interval, $0.03-0.90 ; P=0.04)$.

\section{Discussion}

In the present study, we developed a HF self-care program for Japanese patients delivered by a multidisciplinary team prior to hospital discharge. The new program did not impact on overall HF self-care that was the primary outcome, but one specific behavior regarding a low-salt diet improved, compared with baseline. Moreover, the new program tended to improve patient's HF knowledge over 6 months. Patients in the intervention group had better HF knowledge shortly after discharge. However, the superiority in knowledge in the intervention group faded out at the sixth month. A marginal significance in HF hospitalization/cardiac death in the intervention group was observed. In view of these results, impacts of the new HF self-care program would be limited. However, these drawbacks could provide a direction for future trials. We discuss here lessons learnt from this randomized controlled pilot study.

There were no differences in HF self-care behavior, despite observing a trend of improvement in HF knowledge 


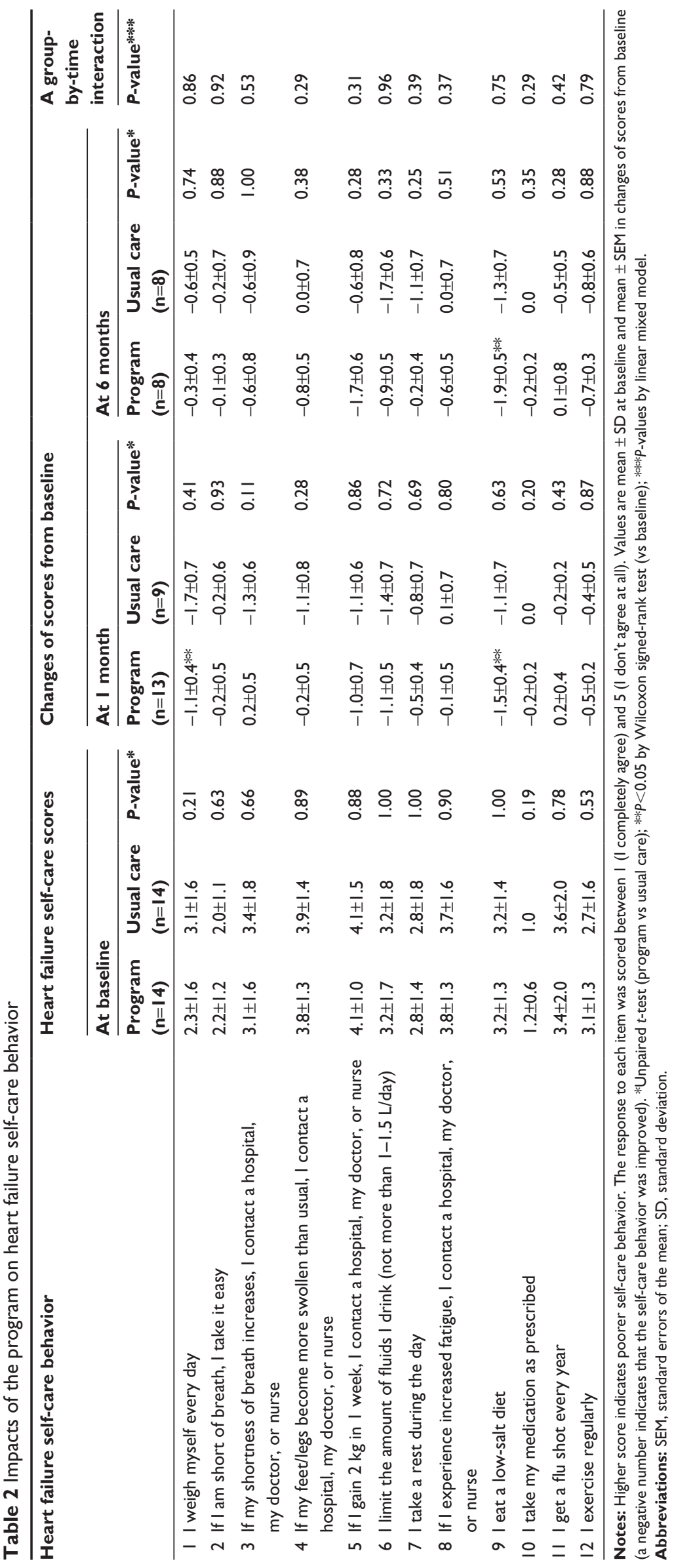




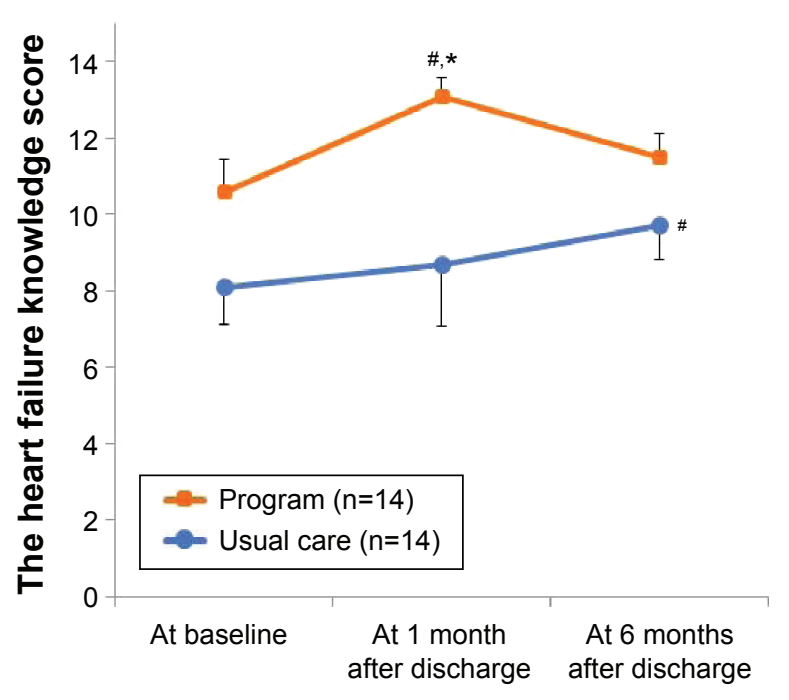

Figure 4 Impacts of the program on heart failure knowledge.

Notes: Data represents mean of the heart failure knowledge score and error bars represent standard errors of the mean (SEM). Higher score indicates greater knowledge about heart failure (range: $0-15)$. Follow-up time $(F=5.23, P<0.01)$, group $(F=6.89, P=0.01)$, and a group-by-time interaction $(F=2.47, P=0.098)$ by the linear mixed model. ${ }^{\# P}<0.05$ by unpaired $t$-test (the intervention group vs the usual care group); ${ }^{*} P<0.05$ by paired $t$-test (vs baseline).

in the intervention group compared with the usual care group over 6 months. These results confirm that merely increasing HF knowledge of patients does not always contribute to a change in their behavior. This may be attributable to the fact that our HF program puts a little more emphasis on increasing HF knowledge than on developing the skills that support self-care in unique situations. ${ }^{23}$ Further improvement focused on self-care skills in changing behavior (such as how to develop an exercise plan) is necessary. ${ }^{24}$

Our results confirm the need for continuous self-care support. ${ }^{6} \mathrm{HF}$ patients in both groups improved their self-care behavior at 1 month after discharge, but these beneficial effects decreased at 6 months after discharge. Although our study patients were followed up every $1-2$ months by cardiologists or attending physicians, more structured attention to self-care over a longer period in an outpatient clinic may be needed for our patients. HF patients sometimes face difficulty in incorporating self-care behavior into their daily lives after discharge. It may be difficult to provide enough education about self-care skills during their hospitalization alone. In addition, education needs to be repetitive and reinforced regularly to promote patient self-care competence and confidence. Since Japanese hospitals cannot receive reimbursement for nurse-led education and counseling for HF patients at outpatient clinics, this would be difficult for nurses to put in practice. These findings emphasize the need for modifying our Japanese health care system. Postdischarge support by HF nurses may be effective in Japan, as in Western countries. .,6,25 $^{-}$

Interestingly, we observed a beneficial effect on sodiumrelated knowledge and self-care behavior in the intervention group. Such a behavioral change is very important especially in Japan because the average salt intake of Japanese adults is relatively high (10.2 $\mathrm{g}$ /day, 4,017 $\mathrm{mg}$ sodium) compared with Western people (eg, Americans consume $8.2 \mathrm{~g}$ /day, $3,400 \mathrm{mg}$ sodium), ${ }^{26,27}$ and adherence to a low-salt diet was not sufficient in Japan. ${ }^{22,28}$ Non-adherence to salt and water restriction were the leading causes of rehospitalization in Japanese patients with $\mathrm{HF},{ }^{29}$ which may explain one of the potential reasons for the reduction of HF hospitalization and/or cardiac death in this study. ${ }^{30}$ A study revealed that the frequency of delivering self-care education with regard to a low-salt diet was low (48\%) among hospital's nurses. ${ }^{31}$ Participation of a dietician in the HF multidisciplinary education is recommended by the HF guidelines; ${ }^{16}$ however, in real-world clinical practice, dietician involvement is often limited to patients with nutritional problems such as obesity, diabetes, or renal disease. In our study, all patients in the intervention group received dietary education by a dietician as well as by nurses. In a prior study, nurse-delivered education was not related to the dietary sodium knowledge score, ${ }^{13}$ whereas dietician education improved adherence to a sodium-restricted diet in a randomized trial. ${ }^{32}$ These results may indicate the benefits of education and counseling delivered by dieticians.

Consistent with earlier studies, ${ }^{33,34}$ the new HF program prior to hospital discharge was likely to reduce the rate of HF hospitalization and/or cardiac death. The benefits of the program may be attributable to increased HF knowledge as well as behavior change with regard to a low-salt diet as described earlier. Several studies have also reported that the enhancement of knowledge is related to a reduced risk of hospitalization. ${ }^{13,14}$ Increased knowledge does not always guarantee good adherence to self-care behavior as seen in our study; however, HF knowledge is vital for better outcomes as shown in previous studies. ${ }^{12,13}$ In fact, hospitalization for worsening $\mathrm{HF}$ is sometimes caused by inadequate patient knowledge about diet, medication, and HF symptoms. ${ }^{12,35}$

On the one hand, the improvement of HF knowledge was short term and did not last long in the present study. One may argue that this transient improvement of HF knowledge could really lead to the reduction of HF hospitalization/cardiac death in this study. A recent Japanese study has suggested that an intensive education in the hospital reduced HF hospitalizations and mortality among Japanese HF patients. ${ }^{36}$ 


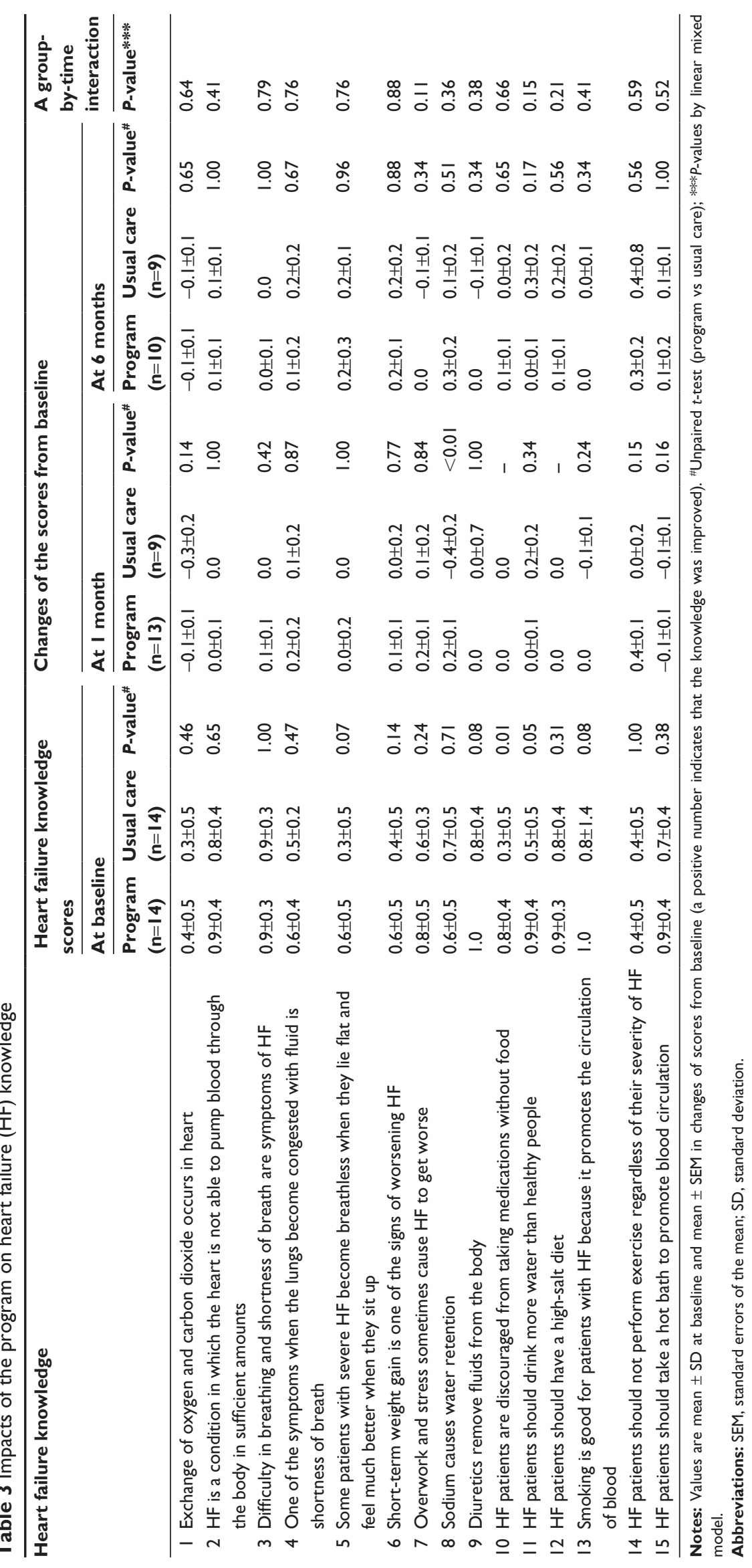




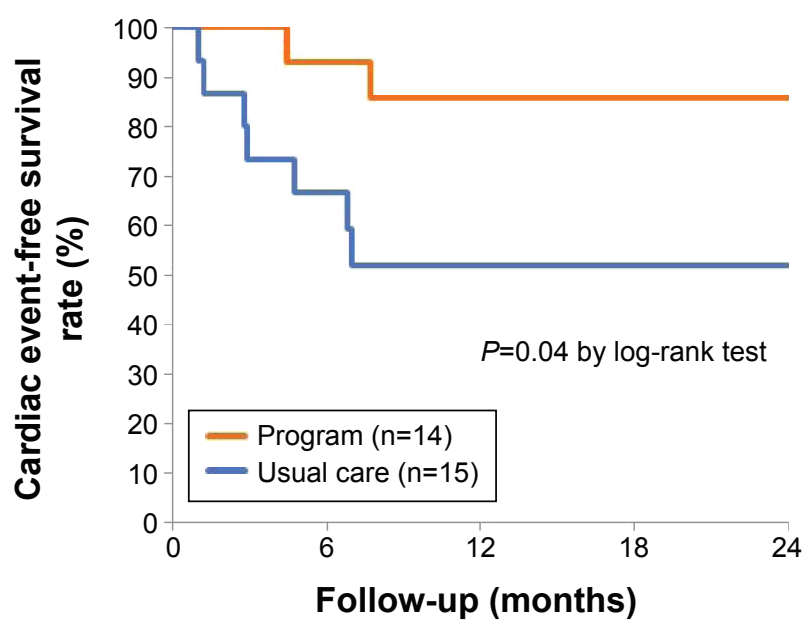

Figure 5 Impacts of the program on heart failure hospitalization and/or cardiac death.

Most of the rehospitalization occurs immediately after discharge, ${ }^{37}$ and a high prevalence of cardiac events within 6 months was also found in the present study. Although the effect of the educational program in the hospital is limited, it is likely to be effective for preventing at least early rehospitalization after discharge.

\section{Implications for research and lessons learnt for a Japanese setting}

The new HF program was likely to increase patients' HF knowledge and change their behavior with regard to a low-salt diet and it is likely to reduce hospitalization for HF. This simple education program would be easy to apply in other medical institutions. On the other hand, some parts of this program should be changed on the basis of what we have learnt from this pilot study. In particular, the program should be modified to focus on self-care skills so that patients can turn their knowledge into actions. Although HF patients are regularly followed up by their physicians, our findings suggest an additional need for continuous self-care support. Further research is necessary to examine the impacts of a HF program that is combined with self-care support after discharge in a Japanese setting.

\section{Study limitations}

One of the major limitations of this study was the small sample size. First, although we referred to a prior study by Lupon ${ }^{21}$ to calculate sample size, the effect size may be relatively larger. The interpretation of the results should be conservative. Second, the intervention was conducted in a single hospital. However, the relative simplicity of our HF self-care program should make it easily applicable to a broad spectrum of patients with HF. Third, we evaluated the impacts of the program on HF self-care behavior only at 1 and 6 months. The long-term effect of the program on HF self-care behavior at 12 months remains unknown. To overcome these limitations and confirm the generalizability of results in the present study, a large multicenter study with long-term follow-up is necessary in the future.

\section{Conclusion}

We developed an HF self-care program for Japanese patients delivered by a multidisciplinary team before hospital discharge. The HF program is likely to improve patients' HF knowledge, change their behavior with regard to a low-salt diet, and reduce rehospitalization for worsening HF and/or cardiac death. However, there was no significant difference in the overall HF self-care assessed by the total EHFScBS score between the intervention group and the usual care group. Future improvements of this program are necessary, focused on facilitating the development of self-care skills. Further research is needed to develop a combination program of this program and postdischarge support.

\section{Acknowledgment}

This work was supported in part by a Grant-in-Aid for the Postdoctoral Research Fellowship from the Japan Society for the Promotion of Science (JSPS) to N.P.K. (no 224943).

\section{Disclosure}

The authors report no conflicts of interest in this work.

\section{References}

1. Okura Y, Ramadan MM, Ohno Y, et al. Impending epidemic: future projection of heart failure in Japan to the year 2055. Circ J. 2008;72(3): 489-491.

2. Riegel B, Moser DK, Anker SD, et al. State of the science: promoting self-care in persons with heart failure: a scientific statement from the American Heart Association. Circulation. 2009;120(12):1141-1163.

3. Jaarsma T, Stromberg A. Heart failure clinics are still useful (more than ever?). Can J Cardiol. 2014;30(3):272-275.

4. McAlister FA, Stewart S, Ferrua S, McMurray JJJV. Multidisciplinary strategies for the management of heart failure patients at high risk for admission: a systematic review of randomized trials. J Am Coll Cardiol. 2004;44(4):810-819.

5. Wakefield BJ, Boren SA, Groves PS, Conn VS. Heart failure care management programs: a review of study interventions and meta-analysis of outcomes. J Cardiovasc Nurs. 2013;28(1):8-19.

6. Thomas R, Huntley A, Mann M, et al. Specialist clinics for reducing emergency admissions in patients with heart failure: a systematic review and meta-analysis of randomised controlled trials. Heart. 2013; 99(4):233-239.

7. Takeda A, Taylor SJ, Taylor RS, Khan F, Krum H, Underwood M. Clinical service organisation for heart failure. Cochrane Database Syst Rev. 2012;9:Cd002752.

8. Kato N, Makaya M, Ikegame T, et al. Treatment and care for outpatients with feart failure: a nationwide survey (in Japanese). J Jpn Cardiovasuc Nurs. 2012;8(1):68-70. 
9. Kato N, Kinugawa K, Nakayama E, et al. Insufficient self-care is an independent risk factor for adverse clinical outcomes in Japanese patients with heart failure. Int Heart J. 2013;54(6):382-389.

10. van der Wal MHL, van Veldhuisen DJ, Veeger NJGM, Rutten FH, Jaarsma T. Compliance with non-pharmacological recommendations and outcome in heart failure patients. Eur Heart J. 2010;31(12): 1486-1493.

11. Riegel B, Lee CS, Dickson VV. Self care in patients with chronic heart failure. Nat Rev Cardiol. 2011;8(11):644-654.

12. Kollipara UK, Jaffer O, Amin A, et al. Relation of lack of knowledge about dietary sodium to hospital readmission in patients with heart failure. Am J Cardiol. 2008;102(9):1212-1215.

13. Kommuri NV, Johnson ML, Koelling TM. Relationship between improvements in heart failure patient disease specific knowledge and clinical events as part of a randomized controlled trial. Patient Educ Couns. 2012;86(2):233-238

14. Davis KK, Mintzer M, Dennison Himmelfarb CR, Hayat MJ, Rotman S, Allen J. Targeted intervention improves knowledge but not self-care or readmissions in heart failure patients with mild cognitive impairment. Eur J Heart Fail. 2012;14(9):1041-1049.

15. Kato N, Kinugawa K, Sano M, et al. Development of self-care educational material for patients with heart failure in Japan: a pilot study. Nurs Health Sci. 2012;14(2):156-164.

16. The Japanese Circulation Society. Guidelines for treatment of chronic heart failure (JCS 2010). 2010; Available from: http://www.j-circ.or.jp/ guideline/pdf/JCS2010_matsuzaki_h.pdf. Accessed May, 20, 2015.

17. Janz NK, Champion VL, Strecher VJ. The health belief model. In: Glanz K, Rimer BK, Lewis FM, editors. Heath Behavior and Health Education: Theory, Research and Practice. 3rd ed. San Francisco: Jossey-Bass; 2002:45-66.

18. Kato N, Ito N, Kinugawa K, Kazuma K. Validity and reliability of the Japanese version of the European Heart Failure Self-Care Behavior Scale. Eur J Cardiovasc Nurs. 2008;7(4):284-289.

19. Jaarsma T, Stromberg A, Martensson J, Dracup K. Development and testing of the European Heart Failure Self-Care Behaviour Scale. Eur J Heart Fail. 2003;5(3):363-370.

20. Kato N, Kinugawa K, Nakayama E, et al. Development and psychometric properties of the Japanese Heart Failure Knowledge Scale. Int Heart J. 2013;54(4):228-233.

21. Lupon J, Gonzalez B, Mas D, et al. Patients' self-care improvement with nurse education intervention in Spain assessed by the European heart failure self-care behaviour scale. Eur J Cardiovasc Nurs. 2008; 7(1):16-20.

22. Kato N, Kinugawa K, Ito N, et al. Adherence to self-care behavior and factors related to this behavior among patients with heart failure in Japan. Heart Lung. 2009;38(5):398-409.

23. Moser DK, Dickson V, Jaarsma T, Lee C, Stromberg A, Riegel B. Role of self-care in the patient with heart failure. Curr Cardiol Rep. 2012; 14(3):265-275.
24. Dickson VV, Riegel B. Are we teaching what patients need to know? Building skills in heart failure self-care. Heart Lung. 2009;38(3): 253-261.

25. Clark AP, McDougall G, Riegel B, et al. Health status and self-care outcomes after an education-support intervention for people with chronic heart failure. J Cardiovasc Nurs. 2015;30:S3-S13.

26. Japan's Ministry of Health, Labour and Welfare. Results of National Health and Nutrition Survey in 2013. 2013; Available from http://www. mhlw.go.jp/file/04-Houdouhappyou-10904750-Kenkoukyoku-Gantaisa kukenkouzoushinka/0000106403.pdf. Accessed December 14, 2015.

27. Bernstein AM, Willett WC. Trends in 24-h urinary sodium excretion in the United States, 1957-2003: a systematic review. Am J Clin Nutr. 2010;92(5):1172-1180.

28. Jaarsma T, Stromberg A, Ben Gal T, et al. Comparison of self-care behaviors of heart failure patients in 15 countries worldwide. Patient Educ Couns. 2013;92:114-120.

29. Tsuchihashi M, Tsutsui T, Kodama K, Kasagi F, Takeshita A. Clinical characteristics and prognosis of hospitalized patients with congestive heart failure - a study in Fukuoka, in Japan. Jpn Circ J. 2000;64: 953-959.

30. Song EK, Moser DK, Dunbar SB, Pressler SJ, Lennie TA. Dietary sodium restriction below $2 \mathrm{~g}$ per day predicted shorter event-free survival in patients with mild heart failure. Eur J Cardiovasc Nurs. 2014;13(6): 541-548.

31. Albert NM, Cohen B, Liu X, Best CH, Aspinwall L, Pratt L. Hospital nurses' comfort in and frequency of delivering heart failure self-care education. Eur J Cardiovasc Nurs. 2015;14(5):431-440.

32. Arcand JA, Brazel S, Joliffe C, et al. Education by a dietitian in patients with heart failure results in improved adherence with a sodium-restricted diet: a randomized trial. Am Heart J. 2005;150(4):716.

33. Koelling TM, Johnson ML, Cody RJ, Aaronson KD. Discharge education improves clinical outcomes in patients with chronic heart failure. Circulation. 2005;111(2):179-185.

34. Krumholz HM, Amatruda J, Smith GL, et al. Randomized trial of an education and support intervention to prevent readmission of patients with heart failure. J Am Coll Cardiol. 2002;39(1):83-89.

35. Annema C, Luttik ML, Jaarsma T. Reasons for readmission in heart failure: Perspectives of patients, caregivers, cardiologists, and heart failure nurses. Heart Lung. 2009;38(5):427-434.

36. Kinugasa Y, Kato M, Sugihara S, et al. Multidisciplinary intensive education in the hospital improves outcomes for hospitalized heart failure patients in a Japanese rural setting. BMC Health Serv Res. 2014; $14: 351$.

37. Desai AS. The three-phase terrain of heart failure readmissions. Circ Heart Fail. 2012;5(4):398-400.
Patient Preference and Adherence

\section{Publish your work in this journal}

Patient Preference and Adherence is an international, peer-reviewed, open access journal that focuses on the growing importance of patient preference and adherence throughout the therapeutic continuum. Patient satisfaction, acceptability, quality of life, compliance, persistence and their role in developing new therapeutic modalities and compounds to optimize

\section{Dovepress}

clinical outcomes for existing disease states are major areas of interest for the journal. This journal has been accepted for indexing on PubMed Central. The manuscript management system is completely online and includes a very quick and fair peer-review system, which is all easy to use. Visit http://www. dovepress.com/testimonials.php to read real quotes from published authors. 